\title{
FAMILY CORONAVIRIDAE
}

These viruses (100 $\mathrm{nm}$ in diameter) have chracteristically long (12-24 nm), widely spaced, bulbous surface projections (peplomeres), a lipid-containing envelope, and a single-stranded $\mathrm{RNA}^{+}$genome. The virus replicates in the cytoplasm of the infected cell and matures by budding through the intracytoplasmic membranes. Viral inclusion bodies may be seen in the cytoplasm (figure 67): The mechanisms of virus replication are similar to those described for the picorna- and togavirus families.

Actinomycin D, which inhibits DNA-dependent RNA synthesis, markedly inhibits human coronavirus replication. This means that a host cell component is required during the early stages of virus replication (Kennedy and JohnsonLussenberg 1979).

\section{Genus Coronavirus (from corona, meaning crown)}

The virions that have the ability to hemagglutinate red blood cells include:

Avian infectious bronchitis virus (IBV)

Calf neonatal diarrhea coronavirus (figure 67)

Feline infectious peritonitis virus

Human coronavirus

Murine hepatitis virus (MHV), strain JHM 


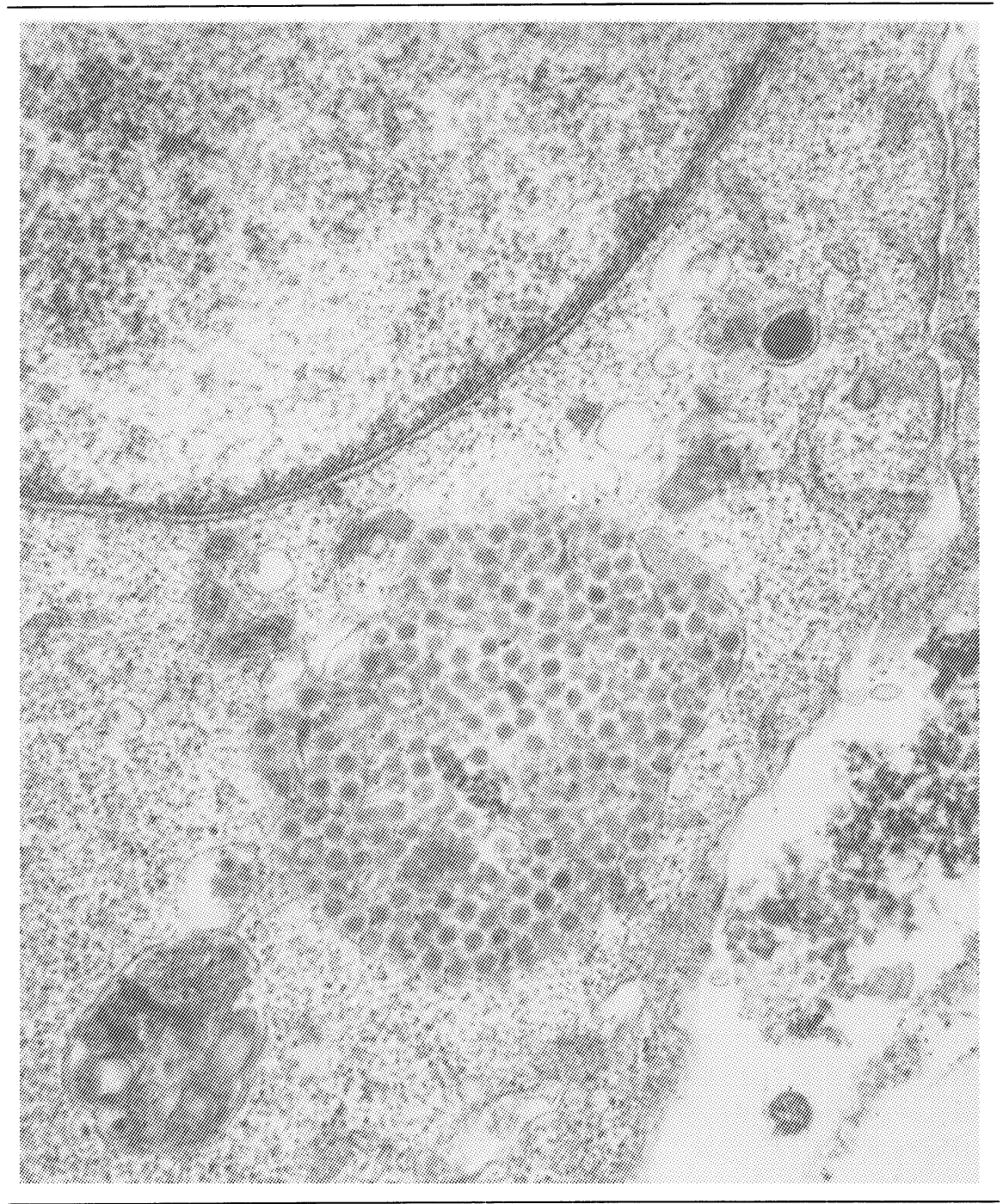

Figure 67. Electron micrograph of a cell infected with a bovine coronavirus $(\times 24,000)$. (Courtesy of Dr. Daniel Dekegel, Institut Pasteur du Brabant, Brussels, Belgium.)

Porcine transmissible gastroenteritis virus (TGEV) Rat coronavirus

Turkey bluecomb disease virus

\section{RNA and proteins}

The RNA of several coronaviruses is polyadenylated and infectious and probably acts as mRNA. An infectious RNA of about $8 \times 10^{6}$ daltons, with cova- 
lently attached polyadenylic acid sequences, has been extracted from avian coronavirus (Lomniczi and Kennedy 1977). Molecular weights of $5.4 \times 10^{6}$ and $3.8 \times 10^{6}$ were reported for RNA genomes of MHV and bovine coronavirus, respectively (Lai and Stohlman 1978; Guy and Brian 1979).

Purified coronavirus JHM contains six polypeptides: GP1, GP2, GP3, VP4, GP5, and VP6, with apparent molecular weights of 170,000; 125,000; 97,500; 60,800; 24,800; and 22,700, respectively. GP2 and GP3 probably protrude from the lipid envelope and together with GP1 form the spike layer. Protein VP6 and part of GP5 are located within the lipid bilayer (Wege et al. 1979). Nine intracellular virus-specific proteins were identified by Bond and associates (1979), of which four were structural proteins. There is a major nonglycosylated protein of molecular weight 50,000-60,000 located inside the virion that is probably the nucleocapsid protein.

\section{DISEASES CAUSED BY THE VIRUS}

\section{Transmissible gastroenteritis (TGE) in pigs}

Infection of the small intestine of the pig occurs after ingestion of food containing virus and leads to destruction of the epithelial cells. After the viremic stage, the virus invades the kidneys and lungs. There are virulent and attenuated virus strains. Young pigs exposed to the virulent virus strains usually die within ten days after exposure.

The problem with producing a vaccinal strain of TGE lies in developing a virus that will stimulate an IgA response in the gut of the sow without producing disease in young pigs (Saif and Bohl 1979).

\section{CNS infection}

Murine coronavirus strain JHM causes demyelination in the CNS in infected mice and rats. This virus normally produces a rapidly fatal encephalomyelitis. However, under certain conditions (such as low virus dose or selection for ts mutants) nonfatal demyelination can be produced due to a selective destruction of the myelin-synthesizing oligodendrocytes (Haspel et al. 1978; Nagashima et al. 1978).

\section{BIBLIOGRAPHY}

Bond, C.W.; Leibowitz, J.L.; and Robb, J.A. Pathogenic murine coronaviruses. II. Characterization of virus-specific proteins of murine coronaviruses JHMV and A59V. Virology 94:371-384, 1979.

Guy, J.S., and Brian, D.A. Bovine coronavirus genome. J. Virol. 29:293-300, 1979.

Haspel, M.V.; Lampert, P.W.; and Oldstone, M.B.A. Temperature-sensitive mutants of mouse hepatitis virus produce a high incidence of demyelination. Proc. Natl. Acad. Sci. USA 75:40334036, 1978.

Kennedy, D.A., and Johnson-Lussenburg, C.M. Inhibition of coronavirus 229E replication by actinomycin D. J. Virol. 29:401-404, 1979.

Lai, M.M.C., and Stohlman, S.A. RNA of mouse hepatitis virus. J. Virol. 26:236-242, 1978.

Lomniczi, B., and Kennedy, I. Genome of infectious bronchitis virus. J. Virol. 24:99-107, 1977. 
Nagashima, K.; Wege, H.; and ter Meulen, V. Early and late CNS-effects of coronavirus infection in rats. Adv. Exp. Med. Biol. 100:395-409, 1978.

Saif, L.J., and Bohl, E.H. Passive immunity in transmissible gastroenteritis of swine: immunoglobulin classes of milk antibodies after oral-intranasal inoculation of sows with a live low cell culture-passaged virus. Am. J. Vet. Res. 40:115-117, 1979.

Wege, H.; Wege, H.; Nogashima, K.; and ter Meulen, V. Structural polypeptides of the murine coronavirus JHM. J. Gen. Virol. 42:37-47, 1979.

\section{RECOMMENDED READING}

Horzinek, M.C., and Osterhaus, A.D.M.E. The virology and pathogenesis of feline infectious peritonitis. Brief Review. Arch. Virol. 59:1-15, 1979. 